\title{
Design and Simulation of Message Display on LCD Integrated with Power Line Carrier Communication Modem with Biometrics System
}

\author{
Purnendu Shekhar \\ Pandey \\ ECE Department \\ THDC IHET , Tehri \\ Uttrakhand, India
}

\author{
Nitin Gupta\& Ajay Raturi \\ Electrical Engg.Department \\ THDC IHET , Tehri \\ Uttrakhand, India
}

\author{
Banit Negi \\ Computer Science Department \\ THDC IHET, Tehri \\ Uttrakhand, India
}

\begin{abstract}
This paper serves a new technology for communication field. As we know that in this era many of the research is going on in communication filed .Main motto of the researcher is to reduce the interference and loss etc. In this paper we developed a circuit which can be helpful to sending the data via exiting wire line (220-230 AC) with very low power consumption and very low data losses in optimum cost. Yet we facing few problems in hill area where it is very tough to align the tower of transmitting signal (provided by the different service provider).As concern to cost it is not possible to install tower or repeater (cost of repeater is approx. $1000 \$$ ) everywhere in such type of area .It is possible to install such type of modem where it is not possible to send the data wirelessly or in high expenditure. We are sending the message signal via exiting power line. Communications over Power line is different than communication over existing networks (for example, UTP, STP, Fiber etc.)Power line communication previously tough medium for transmitting the data. Transmission over the AC line is difficult because of the impulsive noise and interference caused from many sources. Also, the power line is not constant over every time. But as in compared to the Ethernet cabling, which is clean and has consistent characteristics, the power line can have numerous appliances and equipment plugged in at any time we can turned on or off and run for any extent of time. In this paper the modem used is made by sunrom-1187 is based on much reduction in term of error in data reception. In this paper Transmission is based on byte by byte basis. For AC $50 \mathrm{~Hz}$ system the zero crossing of AC signals happens every $10 \mathrm{~ms}$ and modem needs 50 zero crossings to transmit one byte with error checking data. We can give the input with via mobile or keyboard. For security propose we can integrate this circuit with biometric system. We can send the data with keyboard, keyboard decoder decode the input and then it will connect to At mega 32 controller and then program it and simulated with ISP connector and then at last connect Power line Carrier Communication Modem system to the AC power plug. As a result circuit consist of 5 Volt Power supply, At mega 32 controller, ISP Connector, biometric system.
\end{abstract}

\section{Keywords}

LCD, PLCC Modem, Biometric system

\section{INTRODUCTION}

In the current scenario of communication techniques, the present focus is both on formation as well as spreading of information with very less distortion and secure data.We also always kept in our mind that our communication installation should not take very high cost in order to be able to reach the end users for the provision of information, the popular technologies currently being used include telephone wires, Ethernet cabling, fiber optics, wireless personal area networks and satellite technologies. However each has its limitations of cost and availability to reach the maximum number of users with error free data. As we developed the circuit which using the exiting power cable of home so that its cost is very low (approx. 150 \$ only).With biometric system we are making our system safe ,we can also use RFID system instead of this .This Modem having very low power consumption. This modem having the main features like Transmitting and Receiving the serial data at 9600 baud rate and Operating power $5 \mathrm{~V}$.The figure 1 below is showing how we are sending the data through our circuit. We are connected the biometrics system on serial port of USB, decoder decoding the data.

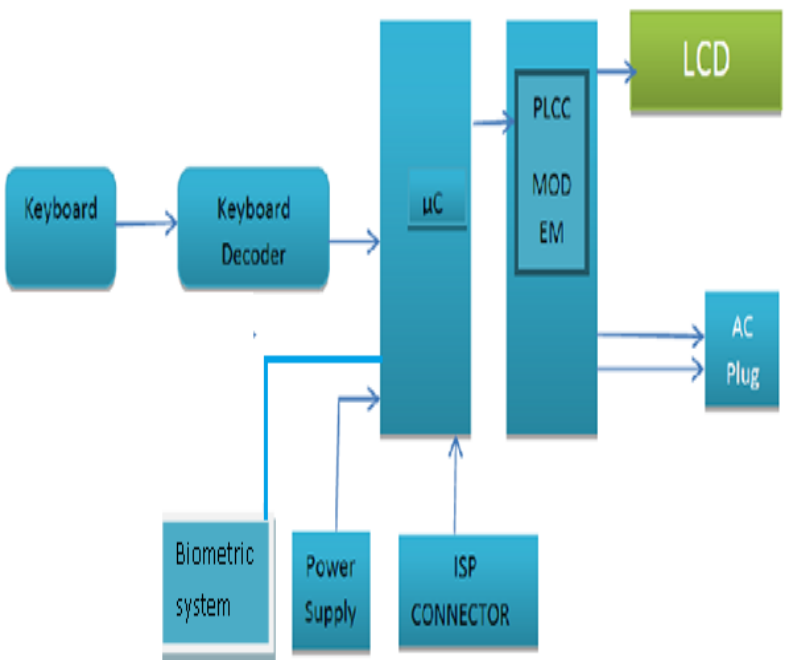

Figure 1: Transmitting end of the circuit

biometric system is mainly for security propose ,as we are reducing the password type security. At receiving end we integrated large LCD to display system to display the message sending from transmitter end as shown in figure 2 below. 


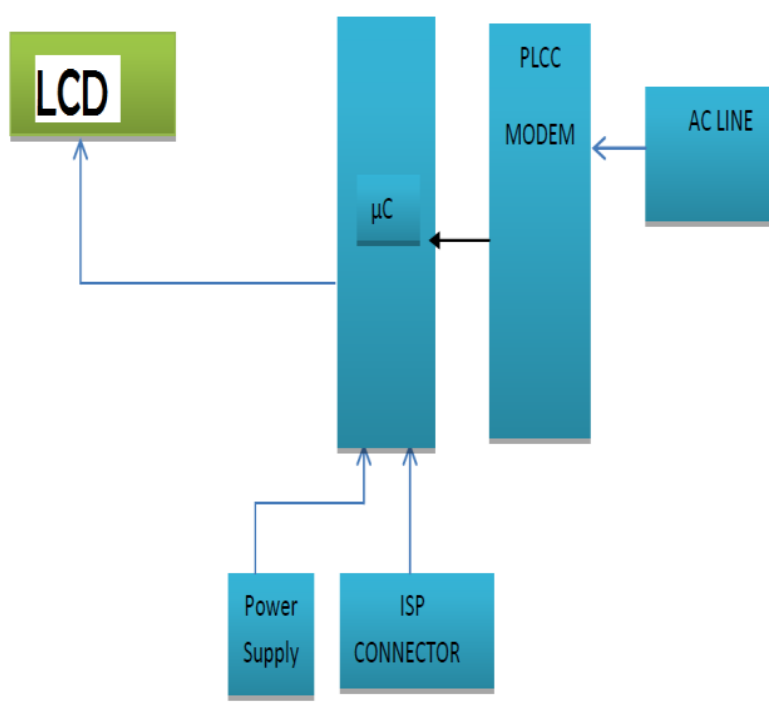

Figure 2: Receiving end of the circuit

Received data came through the AC Power line to PLCC modem. For AC $50 \mathrm{~Hz}$ frequency system the zero crossing of AC signals happens every $10 \mathrm{~ms}$ and modem needs 50 zero crossings to transmit one byte with error checking data. For reducing the interference modem has OFDM .

\section{HARDWARE DESIGN}

The Hardware employment deals in sketch the diagram on the plan paper according to the application, testing the schematic design over the bread board using the various IC's to find if the design meets the objective, carrying out the PCB layout of the schematic tested on breadboard, finally preparing the board and testing the designed hardware. Hardware development of scrolling message display on led with PLCC modem is divided into two parts.

- Hardware Design for PLCC Transmitter

- Hardware Design for PLCC Receiver

\subsection{Hardware Design for PLCC}

The block diagram of the Master mote can be divided in two sections:

- Interfacing Section of the Components

- Power supply section.

The way of interfacing our system with power supply to the PLCC modem. Firstly the keyboard is connected to the keyboard decoder that decodes every bite of data which is then attached to the microcontroller's Rx pin that sends the data to PLCC modem through the controller TX pin, and finally to the $\mathrm{AC}$ lines. We can initially connecting our system with Biometric module (Model No. 3935, Sunrom Technology) to the Port for security purpose. We are interfacing the biometrics system via serial interface, the system with MCU of 3.3 - 5V power: TD (pin 3 of P1) connects with RXD (receiving pin of MCU), RD (pin 4 of Port 1) connects with TXD (transferring pin of MCU). The upper computer (PC) be in RS-232 mode, please add level converting circuit, like MAX232, between the Module and PC figure 3 below showing the inner circuit of biometric system.

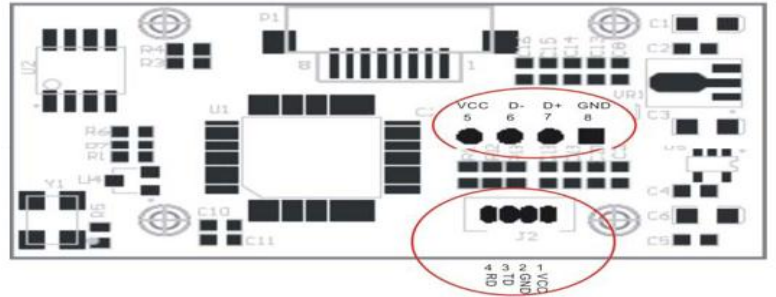

Figure 3 .Inner circuit of biometric System

\subsection{Power Supply Module}

This module is basically designed to achieved $5 \mathrm{~V}$, $500 \mathrm{~mA}$.This consists of a transformer which is used to step down the AC voltage, IN4007 diodes used to form a bridge rectifier to convert $\mathrm{AC}$ to $\mathrm{DC}$,capacitor $1000 \mathrm{uF}$ which used as a filter circuit, 7805 regulator to obtain fixed $5 \mathrm{~V}$ at the output of the regulator , $330 \mathrm{ohm}$ resistance, LED as indicator.

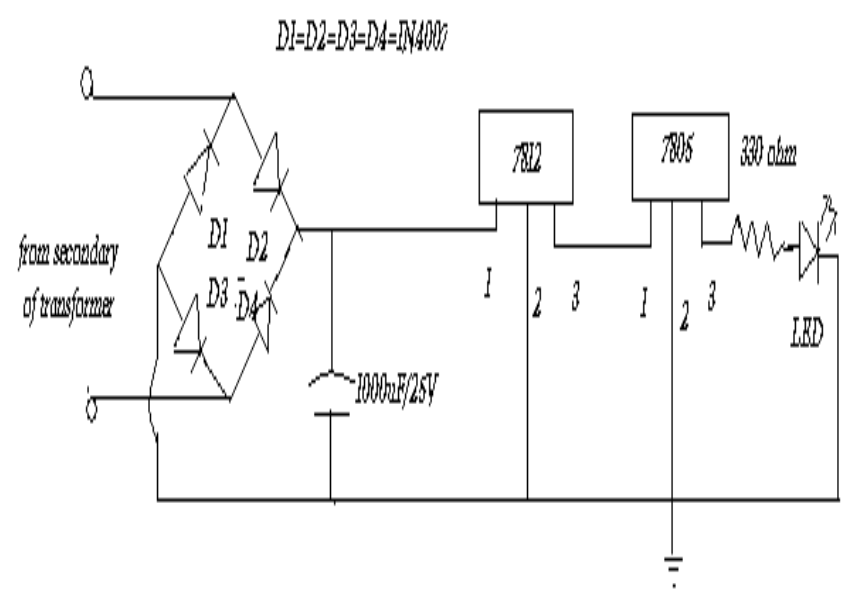

Figure 4 : Circuit of 5 V Power Supply

\subsection{Embedded microcontroller}

There is a whole wide range of microcontroller available in the market. But this particular project is developed using AVR series of microcontroller (ATMEGA 32) because it has Flash memory of $32 \mathrm{~kb}$, SRAM $2 \mathrm{~kb} \&$ EEPROM $1 \mathrm{~kb}$. AT mega 32 is a low-power CMOS 8-bit microcontroller based on the AVR RISC architecture. By executing powerful instructions in a single clock cycle, the AT mega 32 achieves throughputs approaching 1 MIPS per MHz, allowing the system designed to optimize power consumption versus processing speed.

\subsection{Display module}

The LCD(liquid crystal display) unit receives character codes ( 8 bits per character) from a microprocessor or microcomputer, latches the codes to its show data RAM (80byte) DD RAM for storing 80 typescripts, transforms each character code into a $5 \times 7$ dot-matrix character pattern, and displays the characters on its LCD screen. We are $16 \times 2$ LCD's which have 16 columns and 2 rows with 16 hardware pins connected as pin 1,3and 16 are connected to ground, pin 2 and 15 are connected to $+5 \mathrm{v}$ pin $3,4,5$ are RS , RW and enable individually enable pin is always low. Data pins of LCD are $11,12,13,14$ which are used for 4 bit parallel communication. 


\subsection{Transmitting Module (PLCC Modem)}

We used here modem for power line carrier communication made by sunroom technologies, the model number 1187 .Power line modem is useful to send and receive serial data over existing AC mains power lines of the house. It has high protection to electrical noise perseverance in the power line and built in error checking so it never gives out corrupt data. The modem is in form of a ready to use circuit module, which is capable of providing 9600 baud rate low rate bi-directional data communication. Due to its small size it can be integrated into and become part of the user's power line data communication system. It has main features as Transmit and Receive serial data at 9600bps, Data TX/Rx LEDs, Powered from 5V, Low Cost \& Simple to use Built in Error Checking, Direct interface with, microcontroller UART TX/, RX pins. Table 1 shows below interfacing pin detail of PLCC modem.

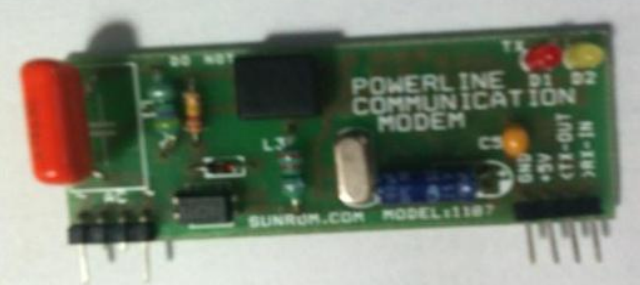

Figure 5 :View of PLCC Modem

\begin{tabular}{|c|c|c|}
\hline Pin & Pin Name & Pin Details \\
\hline $\begin{array}{l}\text { RX- } \\
\text { IN }\end{array}$ & $\begin{array}{l}\text { Receive } \\
\text { Input }\end{array}$ & $\begin{array}{l}\text { Input serial data of } 5 \mathrm{~V} \\
\text { logic level } \\
\text { Usually connected to TXD } \\
\text { pin of microcontrollers }\end{array}$ \\
\hline $\begin{array}{l}\text { TX- } \\
\text { OUT }\end{array}$ & $\begin{array}{l}\text { Transmit } \\
\text { Output }\end{array}$ & $\begin{array}{l}\text { Output serial data of } 5 \mathrm{~V} \\
\text { logic level } \\
\text { Usually connected to RXD } \\
\text { pin of microcontrollers. }\end{array}$ \\
\hline $\begin{array}{l}+5 \\
\text { volt }\end{array}$ & $\begin{array}{l}\text { Power } \\
\text { Supply }\end{array}$ & Regulated 5V supply input. \\
\hline GND & Ground & $\begin{array}{l}\text { Ground level of power } \\
\text { supply. Must be common } \\
\text { ground } \\
\text { With microcontroller. }\end{array}$ \\
\hline
\end{tabular}

Table 1: Interfacing Pin Details of PLCC Modem

\subsection{Interfacing of Biometrics System}

We are interfacing the biometrics system via serial interface, the system with MCU of 3.3 - 5V power: TD (pin 3 of P1) connects with RXD (receiving pin of MCU), RD (pin 4 of Port 1) connects with TXD (transferring pin of MCU). the upper computer (PC) be in RS-232 mode, please add level converting circuit, like MAX232, between the Module and PC.As in figure 7 below .

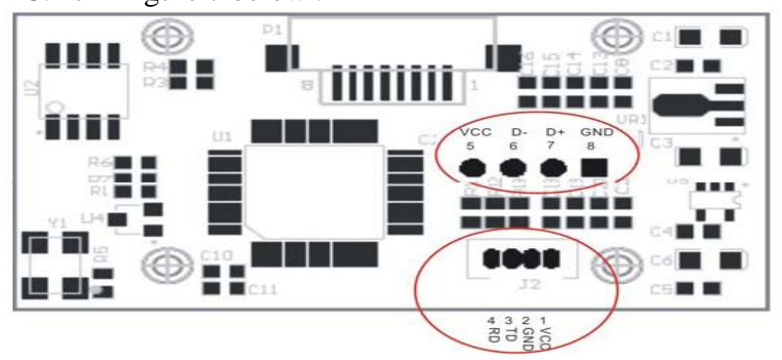

Figure7: Inner Circuit of Biometrics System

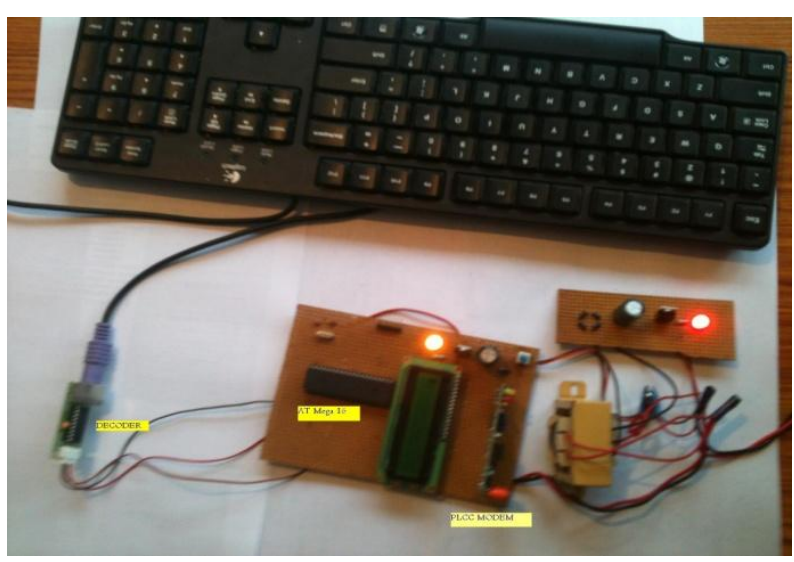

Figure 8: Hardware view of the System

\section{SOFTWARE DEVELOPMENT}

As concern of software development we using proteus software for testing the system .As in Biometric System it's driver is coming with this module we are simply installing the driver through our PC ,connection are mention in Article 2.6.

We initially installing driver of finger print scanner.

Port Control Description of finger print scanner (biometrics system) For UART protocol, it controls the "on/off" of USB port. For USB protocol, it controls the "on/off" of UART port.

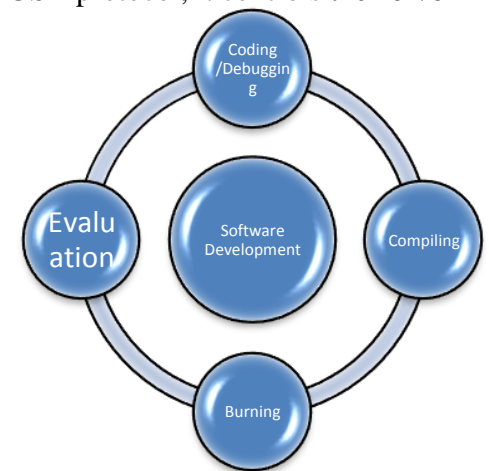

Figure 9: Software Development Process

\subsection{Coding / Debugging}

Figure 9 shows the process of software development, initially we doing coding /debugging process. Coding / debugging in a high-level language (such as C, or Java) or assembler. A compiler for a high level language helps to reduce assembly time. To program the microcontrollers the WinAVR was used. Although inline assembly was possible, the programming was done severely in the $\mathrm{C}$ language. The source code has been observed to facilitate any rare future improvement and maintenance. 


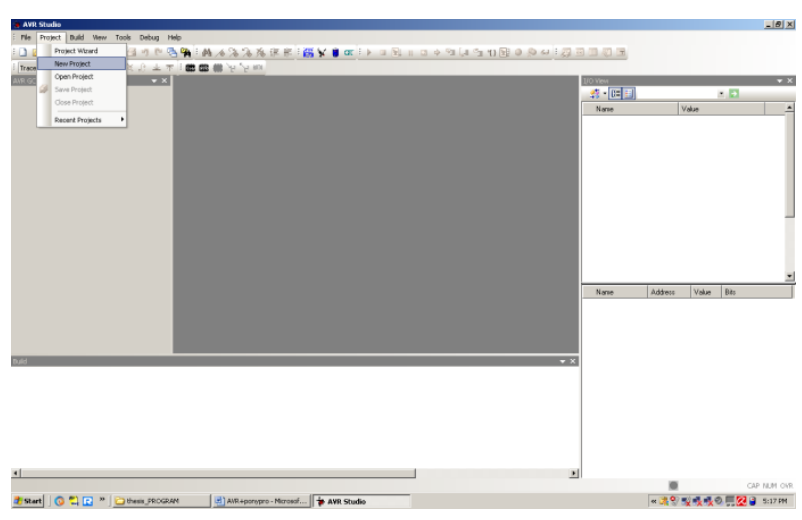

Figure 10: shows the one the process of coding/debugging

We are clicking on the AVR icon and Select new project as shows in figure 10 .

\subsection{Compiling}

The second process of software development is compiling the compilation of the $\mathrm{C}$ program converts it into machine language file (.hex). This is the only language the microcontroller At mega 32 will understand, because it contains the original program code converted into a hexadecimal format. During this step there were some warnings about eventual errors in the program.

\subsection{Burning}

Burning the machine language (hex) file into the microcontroller's program memory is achieved with a dedicated programmer, which attaches to a PC's peripheral. PC's serial port has been used for the purpose. In the present work the Pony Prog programmer has been used to burn the machine language file into the microcontroller's program memory. Figure 11 shows below At mega 32 Programmer (ISP) connections.

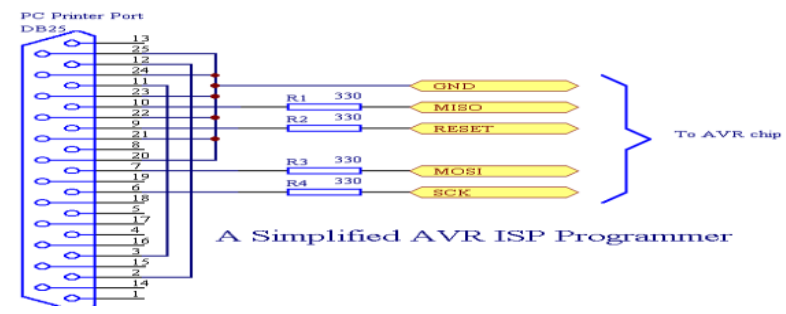

Figure11:Atmega32 Programmer (ISP)

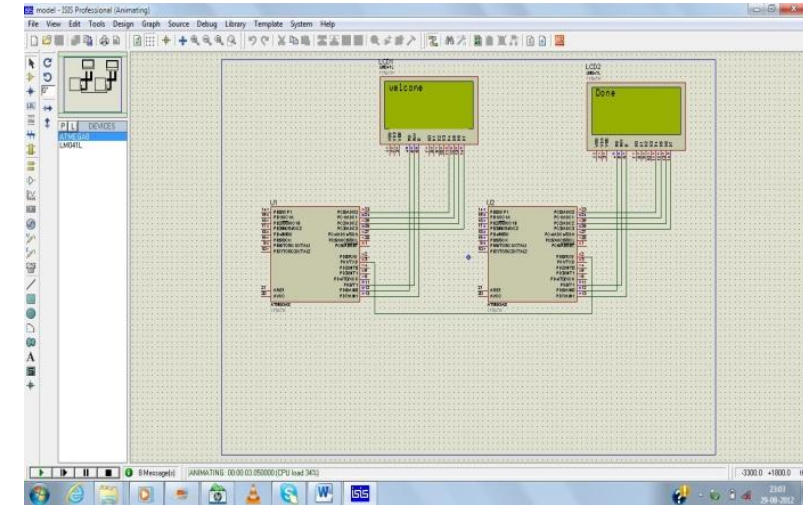

Figure 12: Snap shot of the LCDs connected with controller

\section{CONCLUSION}

Finally we can say this system will be very useful for sending the data in remote areas where the wireless system will not much effected .So use of biometric system in this system we are sending the data in secure ways. It will reduce the interference as concern of PLCC old mythology.

\section{ACKNOWLEDGMENTS}

We are thankful to Dr. Rajesh Singh, Dr. Mayank Singh and Mr.Vivek Kaundal for their guidelines .

\section{REFERENCES}

[1] R.M. Vines et al., "Noise on the residential power distribution circuits", IEEE Transactions on Electromagnetic Compatibility, Vol. 26. No. 4, pp. 161168, November 1984.

[2] FredrikRoos, "PowerlineCommunicationinTrainControlS ystems",MasterThesis,KTH, Stockholm,2000.

[3] "PowerlineTelecommunications(PLT);QualityofService( QoS)requirementsforin-house systems",TechnicalReport,ETSITR102049V1.1.1(200205) 18Tavel, P. 2007 Modeling and Simulation Design. AK Peters Ltd.

[4] Medium-voltage Power Line Carrier Communication System Xiao Hui,Zeng Xangjun, Fan Shaosheng, , Wu Xuebin, and Kuang h g ,2004.

[5] Development of DC Power Line Carrier Communication Interface for Digital Networking for Control of COIL BB Shrivastava, Indian Institute Of Technology, Khargpur 721302. December 20-22,2004.

[6] Power Line Communication Tutorial -Challenges and Technologies Phil Sutterlin and Walter Downey,Echelon Corporation,4015 Miranda Ave, Palo Alto, CA 94304 USA.

[7] DESIGN OF DOMESTIC POWER LINE CARRIER COMMUNICATION,MHM Rikaz, NR Landerz, T Shantharahavan, S Jeyagopikrishna 